\title{
Drug that boosts nerve signals offers hope for multiple sclerosis
}

Trialled antibody treatment thought to work by renewing the protective coating of neurons.

\section{BY HEIDI LEDFORD}

A n experimental antibody drug aimed at protecting nerves from the ravages of multiple sclerosis offers hope for a new way to combat the neurological disease - if researchers can definitively show that it works.

The antibody, anti-LINGO-1, is intended to stimulate regrowth of the myelin sheath, the fatty protective covering on nerve cells that is damaged by multiple sclerosis. Its developer, Biogen of Cambridge, Massachusetts, will present results from a small clinical trial at an American Academy of Neurology meeting this week in Washington DC. If the initial promising results from the trial are confirmed, it will be the first such myelin-regeneration therapy. Other researchers are racing to find more targets and compounds that act similarly.

"Once we get a positive result, the field will move very quickly," says Jack Antel, a neurologist at McGill University in Montreal, Canada. But that excitement is tempered by practical hurdles: there is as yet no proven way to measure remyelination of nerve cells in living humans.

Myelin sheaths insulate and support axons, the fibres that transmit signals between nerve cells. In multiple sclerosis, immune attack destroys these sheaths. Stripped of this protective coating, the axons gradually wither away, causing the numbness and muscle spasms that are characteristic of the disease. The 12 drugs approved in the United States to treat multiple sclerosis slow this immune attack — although sometimes with dangerous side effects. But none stops it, says Bruce Trapp, a neuroscientist at the Cleveland Clinic in Ohio.

Anti-LINGO-1 blocks the LINGO-1 protein, which inhibits the production of myelin. In doing so, the drug spurs myelin growth. It has consistently performed well in animal models and in human cells grown in culture.

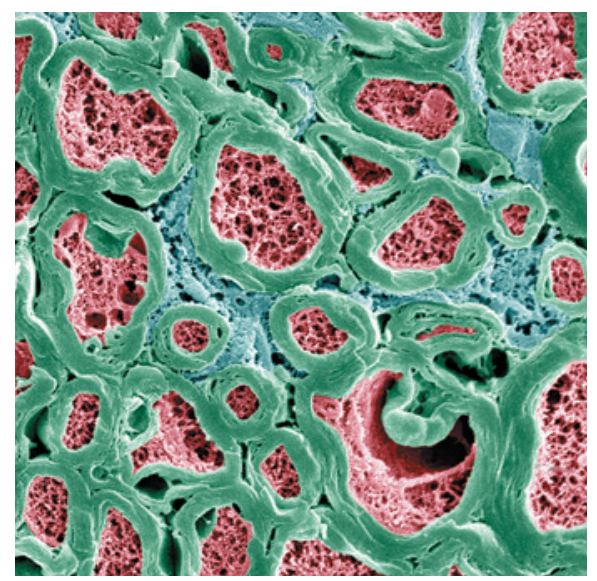

Myelin (green) protects nerve fibres.

LINGO-1 is not the only target for myelinboosting therapies. Acorda Therapeutics of Ardsley, New York, is conducting clinical trials of an antibody that binds to the cells that give rise to myelin, although its molecular target is unknown. And this week in Nature, researchers report that two drugs marketed for skin conditions help to repair myelin in mice and in cultured human cells (F. J. Najm et al. Nature http://dx.doi.org/10.1038/nature14335;2015).

Numerous studies have also found early drug leads, some of them among previously approved therapies. "We're entering the golden age of targets," says Robert Miller, a neuroscientist at George Washington University in Washington DC, who collaborates with Biogen.

Biogen is in the lead, and the industry is watching to see how the company will ascertain whether its drug is working, says Johanne Kaplan, vice-president of neuroimmunology research at Genzyme, a company in Cambridge, Massachusetts, that is also developing remyelinating drugs. Assessing multiple-sclerosis drugs is challenging: symptoms are difficult to measure reliably and often progress slowly. And the benefits of remyelinating drugs are expected to manifest over years, not months.

At the neurology meeting, Biogen will report on a trial of anti-LINGO-1 in 82 patients with optic neuritis, a loss of vision common among people with multiple sclerosis. The trial found that the antibody was no better than a placebo at improving vision. But it did speed up signalling in retinal nerves - a possible sign that the myelin sheath had been rebuilt - by $41 \%$ over placebo after eight months of treatment, says Gilmore O’Neill, vice-president of multiplesclerosis research at Biogen.

The trial was small, and it is too early to know whether the drug will provide tangible relief from other symptoms of multiple sclerosis, such as numbness, cautions Charles ffrenchConstant, who studies the disease at the University of Edinburgh, UK. Similar improvements in nerve conductivity could result from reduced inflammation, he adds. "You can't assume that this is actually evidence of myelin regeneration."

Nevertheless, ffrench-Constant and others are excited to see any sign that the approach may be working. "It is remarkable," says Vittorio Gallo, a neuroscientist at the Children's Research Institute in Washington DC. Biogen is conducting another anti-LINGO-1 trial to test an experimental way (based on magnetic resonance imaging) to assess remyelination, as well as the effect of the drug on symptoms in people for whom the disease is progressing rapidly.

There is little expectation that antiLINGO-1 or other drugs like it will cure multiple sclerosis, says Kaplan. Instead, the hope is that the drugs can be used in combination with the available immune treatments to slow progression of the disease. "If we could get the therapies to patients early enough, maybe we can keep them out of the wheelchair," says Trapp. "That would be huge." -
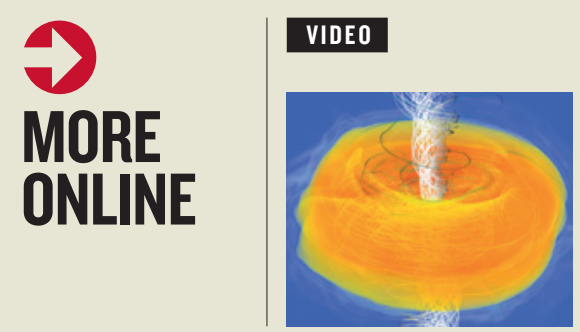

\section{MORE NEWS}

3D models of colliding black holes are most realistic yet go.nature. $\mathrm{com} / 5 \mathrm{vxp} 8 \mathrm{t}$
- US societies push back against NIH reproducibility rules go.nature.com/ $/ 7$ col7 - Shipwrecked 170-year-old champagne gives clues to winemaking's past go.nature.com/cqdwja - Foodies embrace 3D-printed cuisine go.nature.com/8xg1zp

\section{TOP STORY}

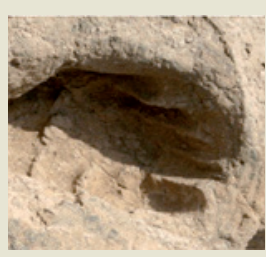

Homo erectus footprints hint at ancient hunting party go.nature.com/ glmonk 